\title{
Material Effective Date Range
}

National Cancer Institute

\section{Source}

National Cancer Institute. Material Effective Date Range. NCI Thesaurus. Code C93860.

The date and time span for when the material is active. 